\title{
Comparison Between Two Warm Ischemic Models in Experimental Liver Transplantation in Pigs
}

F.M. Regueira, A. Espí, P. Nwose, A. Díez-Caballero, J. Baixauli’, F. Rotellar, J. Olea, F. Pardo, J.L. Hernández-Lizoain, and J.A. Cienfuegos

\begin{abstract}
Background. Experimental models of warm ischemia in liver transplantation have been employed to study the mechanisms and treatment of ischemia reperfusion injury.

Material. We compared a control group without (group A, $n=10$ ) versus two models of warm ischemia of liver transplants in pigs: namely, occlusion of the hepatic artery and portal vein for 30 minutes (group B, $n=23$ ) and extraction of the liver 60 minutes after cardiac arrest (group $\mathrm{C}, n=$ 5). Liver function tests, coagulation studies, and liver biopsies were performed during the first 24 hours post-liver transplant.

Results. Clamping of the hepatic vasculature in group B produced a significant liver injury compared with the control group: elevation of the ALT and an abnormal 1-hour postrevascularization biopsy similar to that observed in the cardiac arrest group $\mathrm{C}$. The transaminase levels were lower among group A animals $(P<.05)$. But the hepatic synthetic functions as reflected in the protrombin time (PT) were not affected in group B versus group A. The alteration in PT with respect to the initial value was similar among group A and group B animals, which were significantly less than that in group $\mathrm{C}(P<.05)$.

Conclusion. Occlusion of the hepatic artery and portal vein, a simple surgical maneuver, causes moderate damage to a liver graft but less alteration of hepatic synthetic function. Clamping of the hepatic vasculture obtains more long-term survivors after OLT than cardiac arrest.
\end{abstract}

EXPERIMENTAL MODELS of warm ischemia in liver transplantation have been employed by numerous groups to study the type and mechanisms of ischemiareperfusion injury, to assess the relation of early to eventual function of the allograft, and to test therapeutic options. ${ }^{1-3}$ We compared two models of warm ischemia clamping the hepatic artery and portal vein for 30 minutes, and extraction of the liver 60 minutes after cardiac arrest. Both experimental models have been used by our group in previous studies. ${ }^{4-6}$ The comparisons included a group control in which the graft liver experienced no ischemia.

From the General Surgery Department, University Clinic of Navarra, Pamplona, Spain.

Address reprint requests to F.M. Regueira, General Surgery Department, University Clinic of Navarra, Av Pio XII, No. 36, Pamplona 31008, Spain. E-mail: mregueira@unav.es 


\section{MATERIAL AND METHODS}

Thirty-eight orthotopic liver transplants (OLT) were performed on Large-White pigs. All liver grafts were perfused with $2 \mathrm{~L}$ of University of Wisconsin solution. The liver transplants were performed according to the procedure described by Calne. ${ }^{7}$ The animals were divided into three groups according to the ischemia experienced by the liver donors; Group A or control $(n=10)$ included no extra periods of warm ischemia; Group B or warm ischemia $(n=23)$ included donors subjected to 30 minutes of ischemia before extraction of the liver. Warm ischemia consisted of clamping the hepatic artery and portal vein for 30 minutes (Fig 1). To avoid portal congestion during this time we also clamped the superior mesenteric artery; Group C, included cardiac arrest donors $(n=5)$ wherein the liver was harvested 60 minutes thereafter. Upon laparotomy $90 \mathrm{mg}$ of calcium heparin was administered IV and 10 minutes later, $40 \mathrm{mg}$ of potassium chloride, which caused the animal's death.

At the beginning, at the end, and at 6 and 24 hours post-OLT, blood samples were extracted from the the jugular vein for liver function tests [alanine transaminase (ALT), aspartate transaminase (AST), total bilirubin, lactate dehydrogenase (LDH), alkaline phosphatase as well as coagulation studies including prothrombin time (PT), partial thromboplastine time, thrombin time and fibrinogen]. We performed liver biopsies at the end of the period of ischemia (postischemic biopsy) during the backtable surgery (backtable biopsy) and after revascularization (post-revascularization biopsy). The gradation of preservation lesions was performed according to previously described criteria. ${ }^{8}$ Statistical analysis was performed using SPSS statistical software (SPSS, Inc, Chicago, Ill). Comparing postoperative analytic data using an ANOVA test for repeated means. Qualitative values were assessed using chi-square tests. We considered a difference to be significantly $P<.05$.

\section{RESULTS}

\section{Liver Function}

Group A showed less ischemic injury and better liver function than Groups B and C. The transaminase levels were lower among group A, showing significant improve ment in AST at 24 hours $(P<0.05)$ (Table 1$)$. The evolution of AST was better in Group B than Group C albeit without reaching statistical significance. We did not observe any significant diference in ALT, bilirubin, gamma glutamyl transpeptidase, or alkaline phosphatase values.

\section{Coagulation}

A gradual decrease in prothrombin time was observed in all groups (Table 1). The decrease in PT in relation to the initial value was similar in Groups A (final PT $=62.3 \%$ of initial PT) and B (final $\mathrm{PT}=61.84 \%$ of initial PT), and significantly better than the decreased in group C (final PT = $44.75 \%$ of initial PT; $P<.05$ ). No difference was observed among the values of partial thromboplastine time, thrombin time, and fibrinogen.

\section{Histological Study}

Liver biopsies obtained at the end of the ischemic period of (postischemic biopsy) and during the backtable dissection (backtable biopsy) showed few important lesions; it was similar in the three groups. In contrast, the post-reperfusion biopsies showed focal liver cell necroses with accumulation of neutrophils in many cases. The severity of the preservation lesions in 1 hour post- 
revascularization biopsies was greater for Groups B and C, although no statistical differences were detected between the groups. The gradation of the preservation lesions was: (1) Group A: no lesions in five cases (50\%), mild lesion in three (30\%), moderate lesion in two (20\%); (2) Group B: no lesions in five cases (21.7\%), mild lesion in 10 (43.4\%), moderate lesion in eight (34.78\%); and (3) Group C: no lesions in one cases (20\%), mild lesion in 2 (40\%), moderate lesion in two (40\%).

\section{DISCUSSION}

Warm ischemia may be experimentally reproduced by temporary occusion of the vascular elements of the hepatic pedicle (hepatic artery and/or portal vein) ${ }^{1,2}$ or through cardiac arrest. During the ischemic period the number of blood cellular elements (leukocytes, red blood cells, platelets) in the interior of the vascular structures of the liver are increased, which may contribute to the development of the lesions, this situation may be different from that occuring in clinical transplant, where the interior of the vascular pathway contains preservation liquid composed of cytoprotective elements. ${ }^{9}$ However, the warm ischemic experimental model may reproduce the injury experienced by a marginal liver.

Occlusion of the hepatic artery and portal vein is the method most commonly used in warm ischemic models. ${ }^{1,2}$ Clamping the hepatic artery and the portal vein is a simple surgical maneuver that causes moderate damage to the liver graft. Group B hosts displayed a significant elevation of AST similar to that in Group C; the peak level was statistically significant compared to the control group. But the hepatic synthetic function (showed by the prothrombin time) was less affected in the occlusion than in cardiac arrest group. In fact, a similar pattern in prothrombin time was observed among Groups A and B. Another advantage of clamping of the hepatic vasculature compared with cardiac arrest is to obtain long-term survivors after OLT. ${ }^{45}$ Changes in the 1 hour post-revascularization biopsy were similar among Groups $\mathrm{B}$ and $\mathrm{C}$ hosts. Histological finding were consistent with the observation that an immediate liver biopsy does not predict the function of the hepatic graft. ${ }^{10}$

Although the models of hepatic failure can be easily reproduced it is differences to mimic the conditions of a marginal liver graft. To develop an appropiate model that meets with all the characteristics of chronic liver disease remains an experimental problem. ${ }^{11}$

\section{REFERENCES}

1. Chui C, Toledo-Pereyra L: Transplant Proc 19:1077, 1987

2. Karwinski W, Farstad M, Ulvik R, et al: Transplantation 52:231, 1991

3. Herrera J, Codoceo R, Mora N, et al: Transplant Proc 21:2313, 1982

4. Regueira FM, Herna ndez JL, Sola I, et al: Transplant Proc 29:3347, 1997

5. Nwose PE, Regueira FM, Sierra A, et al: Transplant Proc 31:2431, 1999

6. Espi A, Regueira FM, Toledo G, et al: Transplant Proc 34:3053, 2002

7. Calne R: In Calne R (ed): Liver Transplantation. New York: Grune \& Stratton; 1983

8. Kakizoe S, Yanaga K, Starzl TE, et al: Hepatology 11:932, 1990

9. Belzer F, Southard J: Transplantation 45:673, 1988

10. Starzl T, Demetris A: Current Problems in Surgery 17:51, 1990

11. Mullen K, McCullough A: Hepatology 9:500, 1989. 
Fig 1. Operative field at the beginning (left) and final (right) of 30-minute warm ischemia period (the common hepatic-bulldog champ; portal vein tourniquet).
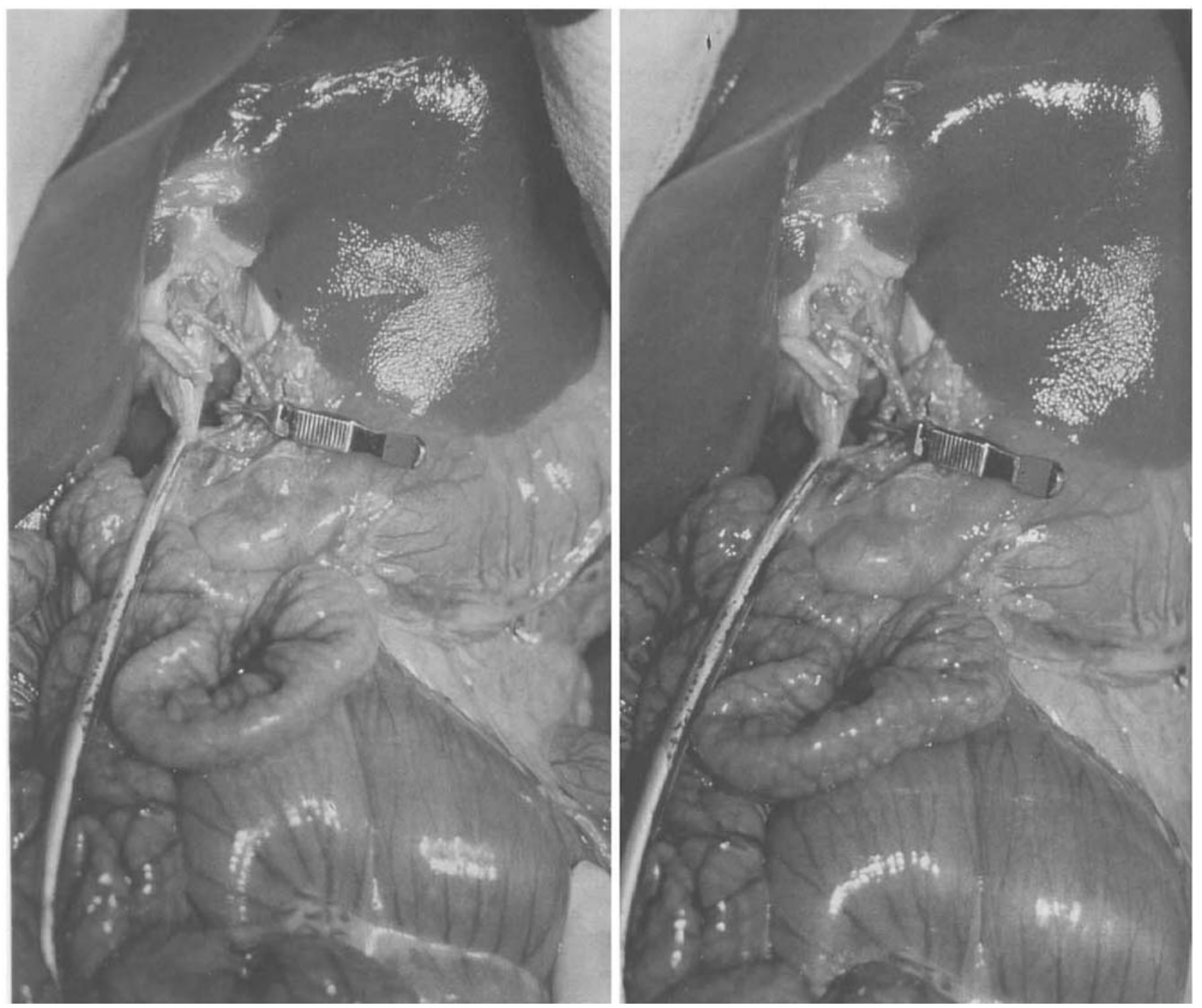
Table 1. Comparison of Alanine Transaminase and Prothrombin Time Between Groups

\begin{tabular}{|c|c|c|c|c|}
\hline & A (Control) $(n=10)$ & $\begin{array}{c}\text { B (Warm Ischemia) }(n= \\
23)\end{array}$ & $\begin{array}{c}\text { C (Cardiac Arrest) }(n= \\
5)\end{array}$ & $P$ \\
\hline \multicolumn{5}{|l|}{ AST (UI/L) } \\
\hline Initial & $40.25 \pm 21.7$ & $39.21 \pm 6.0$ & $31.8 \pm 7.6$ & $<.05^{*}$ \\
\hline Final & $176.56 \pm 72.6$ & $244.29 \pm 73.1$ & $435.8 \pm 192$ & \\
\hline 6 hours & $234.56 \pm 107$ & $478.57 \pm 192$ & $575.6 \pm 226$ & \\
\hline 24 hours & $608.81 \pm 389$ & $1258.55 \pm 737$ & $1319.41 \pm 637$ & \\
\hline \multicolumn{5}{|l|}{$\begin{array}{l}\text { Prothrombin } \\
\text { time (\%) }\end{array}$} \\
\hline Initial & $101.89 \pm 38.5$ & $128.21 \pm 5.5$ & $114.4 \pm 8.7$ & NS \\
\hline Final & $88.78 \pm 10.9$ & $88.61 \pm 10.5$ & $78.3 \pm 8.8$ & \\
\hline 6 hours & $76.01 \pm 13.7$ & $63.35 \pm 14.1$ & $58.04 \pm 10$ & \\
\hline 24 hours & $63.51 \pm 26.7$ & $73.11 \pm 21.7$ & $51.20 \pm 11$ & \\
\hline $\begin{array}{l}\text { PT 24hours/PT } \\
\text { Initial }\end{array}$ & 62.33 & 61.84 & 44.75 & $<.05 * *$ \\
\hline
\end{tabular}

*Control (Group A) warm ischemia (Group B) and cardiac arrest (Group C). **Control (Group A) and warm ischemia (Group B) cardiac arrest (Group C). 